\title{
The CANGAROO-III Project
}

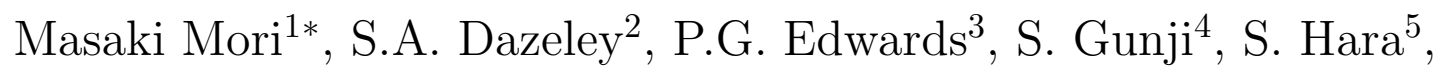 \\ T. Hara ${ }^{6}$, J. Jinbo ${ }^{7}$, A. Kawachi ${ }^{1}$, T. Kifune ${ }^{1}$, H. Kubo $^{5}$, \\ J. Kushida ${ }^{5}$, Y. Matsubara ${ }^{8}$, Y. Mizumoto ${ }^{9}$, M. Moriya ${ }^{5}$,

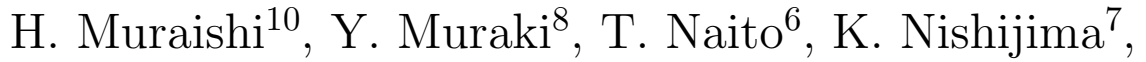 \\ J.R. Patterson ${ }^{2}$, M.D. Roberts ${ }^{1}$, G.P. Rowell ${ }^{1}$, T. Sako ${ }^{8,11}$, \\ K. Sakurazawa ${ }^{5}$, Y. Sato ${ }^{1}$, R. Susukita ${ }^{12}$, T. Tamura ${ }^{13}$, \\ T. Tanimori ${ }^{5}$, S. Yanagita ${ }^{10}$, T. Yoshida ${ }^{10}$, T. Yoshikoshi $^{1}$, and \\ A. Yuki ${ }^{8}$ \\ ${ }^{1}$ Institute for Cosmic Ray Research, University of Tokyo Tanashi, Tokyo 188-8502, Japan \\ ${ }^{2}$ Department of Physics and Mathematical Physics, University of Adelaide, South Australia \\ 5005, Australia \\ ${ }^{3}$ Institute of Space and Astronautical Science, Sagamihara, Kanagawa 229-8510, Japan \\ ${ }^{4}$ Department of Physics, Yamagata University, Yamagata 990-8560, Japan \\ ${ }^{5}$ Department of Physics, Tokyo Institute of Technology, Meguro, Tokyo 152-8551, Japan \\ ${ }^{6}$ Faculty of Management Information, Yamanashi Gakuin Univeristy, Kofu, Yamanashi \\ 400-8575, Japan \\ ${ }^{7}$ Department of Physics, Tokai University, Hiratsuka, Kanagawa 259-1292, Japan \\ ${ }^{8}$ STE Laboratory, Nagoya University, Nagoya, Aichi 464-8602, Japan \\ ${ }^{9}$ National Astronomical Observatory, Tokyo 181-8588, Japan \\ ${ }^{10}$ Faculty of Science, Ibaraki University, Mito, Ibaraki 310-8521, Japan \\ ${ }^{11}$ LPNHE, Ecole Polytechnique. Palaiseau CEDEX 91128, France \\ ${ }^{12}$ Computational Science Laboratory, Institute of Physical and Chemical Research, Wako, \\ Saitama 351-0198, Japan \\ ${ }^{13}$ Faculty of Engineering, Kanagawa University, Yokohama, Kanagawa 221-8686, Japan
}

\begin{abstract}
The CANGAROO-III project, which consists of an array of four $10 \mathrm{~m}$ imaging Cherenkov telescopes, has just started being constructed in Woomera, South Australia, in a collaboration between Australia and Japan. The first stereoscopic observation of celestial high-energy gamma-rays in the $100 \mathrm{GeV}$ region with two telescopes will start in 2002, and the four telescope array will be completed in 2004. The concept of the project and the expected performance are discussed.
\end{abstract}

*) E-mail: morim@icrr.u-tokyo.ac.jp 


\section{INTRODUCTION}

Following the CANGAROO-I $(3.8 \mathrm{~m})$ and CANGAROO-II $(7 \mathrm{~m})$ telescopes, CANGAROO-III is a project to study celestial gamma-rays in the $100 \mathrm{GeV}$ region utilizing a stereoscopic observation of Cherenkov light flashes with an array of four 10-meter telescopes. The CANGAROO-II telescope (hereafter C-II), which has a 7-meter reflector and has been operational since 1999 May [1] [2] [3], is going to be expanded in early 2000 by adding more small mirrors and will be the first $10 \mathrm{~m}$ telescope of this array.

The CANGAROO-III project started in April 1999 and is planned as a five-year program. The schedule is shown in Figure 1. This year we will expand the $7 \mathrm{~m}$ telescope to $10 \mathrm{~m}$, and the second year we will build the second telescope which will be installed in the third year. The other two telescopes will be installed in the fourth and fifth years. Each telescope will be set on a corner of a diamond of about $100 \mathrm{~m}$ side in order to have a maximum number of pairs of telescopes of the same baseline length. The first stereoscopic observation will be performed in 2002 and the full four telescope will be in operation in 2004 .

\section{EXPANSION OF CANGAROO-II}

Expansion of the $7 \mathrm{~m}$ telescope to $10 \mathrm{~m}$ is simple. Since C-II is originally designed as a $10 \mathrm{~m}$ telescope, all we have to do is add 54 mirrors and tune their attitude (Figure 2). This work will be completed in early 2000.

Additional outer mirrors will worsen the point image at the focus but it is not a serious problem. Simulations show that the concentration of photons in one pixel will be reduced from $56 \%$ to $42 \%$ at the center, and from $50 \%$ to $36 \%$ at one-degree

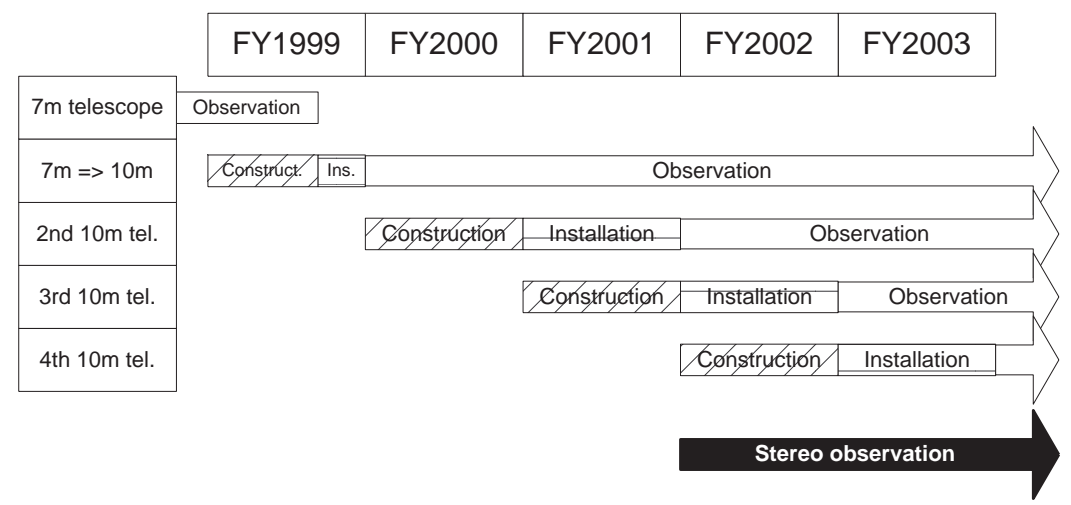

FIGURE 1. Schedule of the CANGAROO-III project. Note that the Japanese fiscal year is from April to March. 
off-axis. In any case, the number of collected photons will be almost doubled, reducing the energy threshold by a factor of two.

\section{CANGAROO-III TELESCOPE DESIGN}

At this stage we will use basically the same design for the support structure and the driving mechanism as the C-II telescope, which is originally designed as a 10-meter telescope and has been proved to work well. The reflector will have a parabolic, composite mirror consisting of 114 small mirrors of $80 \mathrm{~cm}$ in diameter [4]. The focal length will be $8 \mathrm{~m}$ if we use the same mirrors.

Mirrors made of plastic laminates used for C-II are very light and pose little stress on the support structure. Observation of star images at various zenith angles showed the deformation of mirrors was negligible. But the image quality of these mirrors are not as good as glass-made mirrors since they are made by molding: thus we are still investigating other possibilities. The attitude of each mirror will be controlled by stepping motors as for the present $7 \mathrm{~m}$ telescope. Tuning this number of small mirrors to a common focus is not a simple task. For C-II we tuned the mirrors one by one using lids to cover the other mirrors, but this is not easy for larger numbers of mirror segments.

The prime focus camera will be similar to the present CANGAROO-II camera consisting of 512 half-inch photomultipliers and subtending about 3 degrees, but the optimization for stereoscopic observation is underway.

The electronics and data acquisition system will be improved to match higher data rates. In any case, we take timing information of each signal, in addition to pulse height, to utilize the isochronous nature of our parabolic reflector. For stereoscopic observation, we must introduce an inter-telescope trigger to compensate for geometrical delays using programmable delays between telescopes. The local trig-

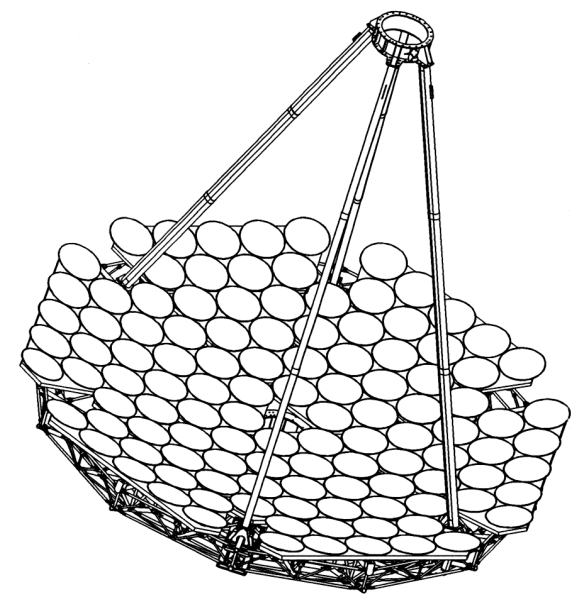

FIGURE 2. A sketch of the $10 \mathrm{~m}$ reflector. 
gers will be as frequent as $1 \mathrm{kHz}$ but the delayed coincidences at the main trigger will be reduced to about $100 \mathrm{~Hz}$, we hope.

\section{STEREO SIMULATION}

Here we briefly show some results of simulations of stereo observations [6]. This work was done before the whole CANGAROO-III project was approved and takes only two telescopes into account, but the result is valid if we use a twofold coincidence in the inter-telescope trigger.

The detection efficiency as a function of baseline length between telescopes is given in Figure 3. If we cut some detected events using the core distance, the energy resolution will be better and an angular resolution less than $0.1^{\circ}$ can be achieved if we use the baseline longer than $100 \mathrm{~m}$. Thus we will adopt the baseline length of around $100 \mathrm{~m}$, which agrees with other calculations. Figure 4 is a comparison of effective area and energy resolution between single and stereo observations.

\section{EXPECTED PERFORMANCE}

Expected sensitivity assuming one $10 \mathrm{~m}$ telescope shown in Figure 5 is around $10^{-12} \mathrm{~cm}^{-2} \mathrm{~s}^{-1}$ above threshold energy of $100 \sim 200 \mathrm{GeV}$, and we may detect many EGRET sources in tens of hours of observation if their spectra extend to higher energies. Also shown are gamma-ray spectra of 22 X-ray selected BL Lacs which are predicted by Stecker et al. [5], however only 5 are in the southern hemisphere.

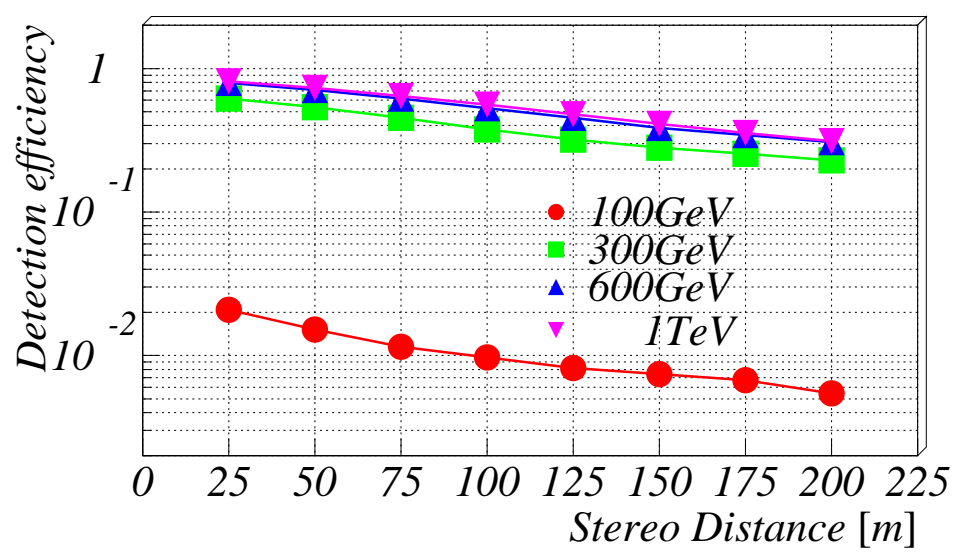

FIGURE 3. Detection efficiency as a function of the baseline separation of two telescopes. Here we define the efficiency as a fraction of triggered gamma-rays when we simulated gamma-rays going vertically and having cores in a circle of $180 \mathrm{~m}$ radius. 
We note that observations at other wavelengths, especially ground-based ones, are rather biased to the northern hemisphere sky and there are undoubtedly more candidate XBLs in the southern sky.

\section{OBSERVATION TARGETS}

Table 1 shows the list of objects observed by the CANGAROO 3.8m telescope for its 6 years of operation. We had been given preference to Galactic sources because of the rather high threshold energy $(\sim 2 \mathrm{TeV})$ of the $3.8 \mathrm{~m}$ telescope, but we may spend more time on extragalactic objects taking account of the lower threshold of new telescopes. One can see from the table we have needed more than 50 hours of observation at least as the necessary condition to conclude "positive detection" with sufficient statistics on the number of gamma-rays. In addition, the imaging Cherenkov technique still suffers from systematic errors which are not negligibly small when compared with gamma-ray signal strength from even "strong sources", and careful estimation on the experimental errors is indispensable by using the data spanning over a long period of observation. We performed survey observations of shorter duration on many sources, which possibly provide a chance of time varying activities of episodic flares, as well as the objects like X-ray binaries which might be "strong sources" if the claims in earlier days are true. The prime efforts of CANGAROO-III will be on those types of objects appearing as top-ranked sources in the table, extending a systematic survey on more sources. In the case of sources of soft spectra, better statistics in the $100 \mathrm{GeV}$ energy region will enable us to detect them in 10 to 20 hours of observation. However, we still have shortage of total observation time available during a year. It is necessary to develop world-wide

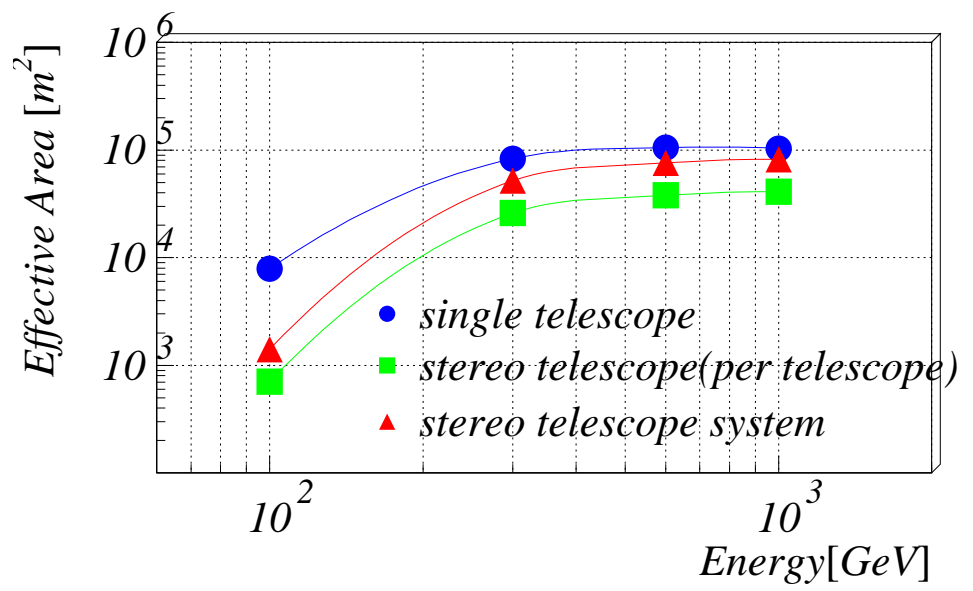

FIGURE 4. Detection area as a function of gamma-ray enegy for single and stereo observations. 
efforts for more new types of high-energy gamma-ray sources in collaboration with other groups proposing next-generation telescopes.

\section{SUMMARY}

CANGAROO-III will start to explore the southern half of the $100 \mathrm{GeV}$ gammaray sky in 2004, complementing projects located in the northern hemisphere to ensure the entire sky is covered at these energies.

\section{REFERENCES}

1. Tanimori, T. et al., Proc. 26th ICRC (Salt Lake City, Utah, USA), OG.4.3.04 (1999).

2. Mori, M. et al., Proc. 26th ICRC (Salt Lake City, Utah, USA), OG.4.3.31 (1999).

3. Kubo, H. et al., in these proceedings.

4. Kawachi, A. et al., in these proceedings; Proc. 26th ICRC (Salt Lake City, Utah, USA), OG.4.3.05 (1999).

5. Stecker, F.W., de Jager, O.C., and Salamon, M.H., Astrophys. J. 473, L75 (1996).

6. Hara, S., Master thesis, Tokyo Inst. Tech. (1999); Hara, S., Kubo, H., and Tanimori, T., in preparation (1999).

7. Kifune, T. et al., Astrophys. J. Lett. 438, L91 (1995).

8. Yoshikoshi, T. et al., Astrophys. J. Lett. 487, L65 (1997).

9. Tanimori, T. et al., Astrophys. J. Lett. 429, L61 (1994).

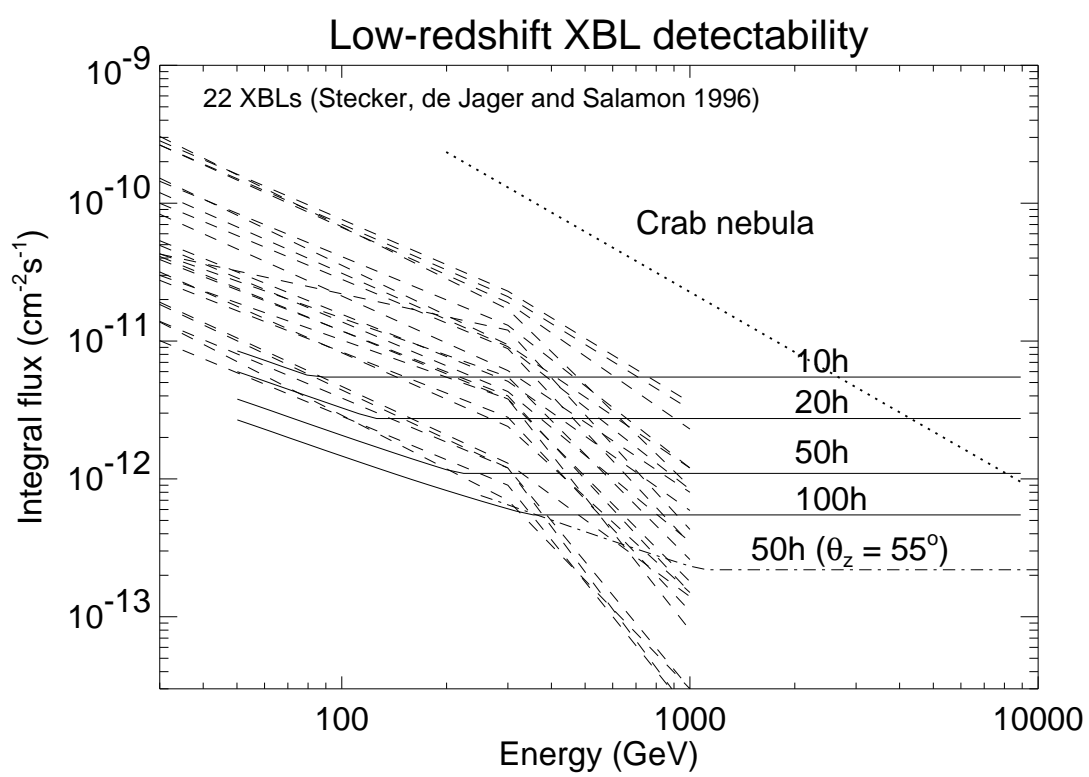

FIGURE 5. Sensitivity of the $10 \mathrm{~m}$ telescope and predicted fluxes from X-ray selected BL Lacs (dashed lines) [5]. Here "10h" means 10 hours of observation and so on. 
10. Tanimori, T. et al., Astrophys. J. Lett. 492, L33 (1998).

11. Sako, T. et al., Proc. 25th ICRC (Durban, South Africa), Vol. 3, 193 (1997).

12. Sako, T. et al., submitted for publication.

13. Mori, M. et al., Proc. 25th ICRC (Rome, Italy), Vol. 2, 487 (1995).

14. Rowell, G. et al., submitted for publication.

15. Roberts, M.D. et al., Astron. Astrophys. 337, 25 (1998).

16. Roberts, M.D. et al., Astron. Astrophys. 343, 691 (1999).

17. Susukita, R., Doctor thesis, Kyoto University (1997) (Preprint KUNS 1450/PN97D09).

18. Rowell, G. et al., Astropart. Phys. 11, 217 (1999).

19. Tanimori, T. et al., Astrophys. J. Lett. 497, L25 (1998).

20. Muraishi, H. et al., Proc. 26th ICRC (Salt Lake City, Utah, USA), OG.2.2.20 (1999).

TABLE 1. A list of objects observed by the CANGAROO $3.8 \mathrm{~m}$ telescope from July 1992 to September 1998 for more than 15 hours in the order of observation time, including bad weather runs. Total observation time is about 2,000 hours. Off-source runs are roughly the same duration but are not listed here.

\begin{tabular}{lcll}
\hline Object & $\begin{array}{c}\text { Observation } \\
\text { time (hr) }\end{array}$ & Remark & Reference \\
\hline PSR1706-44 & 308.5 & Plerion & {$[7]$} \\
Vela & 252.6 & Plerion & {$[8]$} \\
Crab & 193.8 & Plerion & {$[9][10]$} \\
PSR1259-63 & 167.4 & Pulsar binary & {$[11]$} \\
PSR1509-58 & 161.5 & Plerion & {$[11][12]$} \\
W28 & 121.3 & SNR & {$[13][14]$} \\
PKS0521-322 & 104.0 & AGN & {$[15]$} \\
PKS2005-489 & 94.5 & AGN & {$[16]$} \\
Cen A & 80.3 & Radio galaxy & {$[17][18]$} \\
PSR1055-52 & 78.6 & Pulsar & {$[11][17]$} \\
SN1006 & 63.7 & SNR & {$[19]$} \\
RXJ1713.7-394 & 61.4 & SNR & {$[20]$} \\
PKS0548-322 & 49.2 & AGN & {$[16]$} \\
PKS2155-304 & 37.5 & AGN & {$[16]$} \\
PKS2316-423 & 28.8 & AGN & {$[15]$} \\
Sgr A & 28.2 & Galactic center & \\
EXO0423.4-084 & 23.7 & AGN & {$[15]$} \\
GROJ1317-44 & 22.7 & Cen A? & \\
Vela X-1 & 20.8 & X-ray binary & \\
GRB970402 & 19.6 & Gamma-ray burst & \\
Cen X-3 & 17.7 & X-ray binary & \\
2EGJ1746-2852 & 15.2 & EGRET unID & \\
\hline & \multicolumn{3}{l}{}
\end{tabular}

